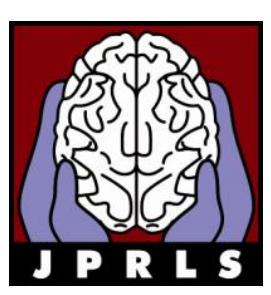

Open Access via www.jprls.org

Journal of Parkinsonism \&

Restless Legs Syndrome

TEACHING

CASE

\title{
Alcohol related fatal brain injury causing multiple types of hemorrhagic lesions
}

\author{
Mohammed A. Rana, ${ }^{1}$ Osama Khan, ${ }^{1}$ Iftikharul Haq, ${ }^{2}$ \\ ${ }^{I}$ Parkinson's Clinic of Eastern Toronto, Toronto, Canada \\ ${ }^{2}$ Dept. of Neurosurgery, Lakehead University, Thunder Bay, Ontario, Canada
}

\begin{abstract}
Traumatic brain injury may be a significant consequence of head trauma, leading to potentially serious disability or death. Although the elderly are especially prone to head trauma due to falls, head injuries and falls related to alcohol use are also quite common and can be seen in younger and middle aged populations. Traumatic brain injury can cause multiple types of cerebral hemorrhagic lesions, which can occur simultaneously. These hemorrhagic lesions can progressively enlarge and cause further neurological deterioration, brainstem herniation, and death. This report highlights a young male with severe brain injury due to considerable alcohol consumption at a party who ultimately died from his injuries. This case report emphasizes the importance of recognizing and raising awareness of the significant role alcohol can play in head related injuries.
\end{abstract}

\section{INTRODUCTION}

Head trauma is one of the leading causes of traumatic brain injury (TBI) that may in turn lead to serious disability and death. The annual incidence of head injuries is about $150-300$ out of 100,000

* Correspondence to: Mohammad A. Rana. Parkinson's Clinic of Eastern Toronto, 741 Broadview Ave, B3, Toronto, Canada M4K2P6. Email: maranajprls@gmail.com. population. ${ }^{1}$ Falls are one of the most common causes of head injury. Alcohol intoxication has been found to be closely related to the incidence of falls. In the elderly population physical illness and alcohol use are strong factors in the occurrence of falls. ${ }^{2}$ Similarly, in the young population, the ingestion of alcohol is also recognized as a common cause leading to fatal outcomes. Secondary brain injury can have a worsening outcome due to the potential presence of cerebral contusion and brain edema. As a consequence, brainstem herniation can occur leading to further neurological deterioration and ultimately death. Studies have shown that alcohol increases the mortality rate among pre-hospital fall victims. This is attributed to the type of fall, height of the fall, type and severity of the injury. ${ }^{3}$ It has been reported that extended alcohol use may also contribute to changes in the brain vascular architecture, which in turn becomes a notable risk factor in causing TBIs such as subarachnoid hemorrhages. ${ }^{4}$ The current recommendations in managing these patients are to assess the risk factors, close clinical monitoring and serial imaging. There are multiple factors which determine the risk of mortality in these cases, namely, age of 45 years or more, alcohol intoxication, GCS of 3-8 at admission, arrival GCS-M of 4 or less, Head- AIS of 4 or more, and admission SBP $<90 \mathrm{mmHg}$. ${ }^{1}$ Head injury due to alcohol consumption continues to be a substantial public health problem, and early intervention is important in these cases. We present a case of a young male who suffered a fatal TBI because of excessive alcohol consumption. 


\section{CASE REPORT}

A 25 year old, previously healthy right handed male was walking down a set of stairs after significant alcohol consumption. He fell down approximately 10 stairs and was found in an inebriated and drowsy state at the foot of the stairs by his peers who were also drinking. Even though they noticed a small laceration on the back of his head instead of seeking hospital attention, his friends thought it was better if he was put to bed due to his intoxicated state. They called for EMS services 8 hours later when he could not be aroused from his sleep.

\section{PHM: None}

Medications: None

Social History: $\mathrm{He}$ worked as a sale representative for an insurance company. He did not smoke cigarettes and consumed alcohol on weekends only.

Family history was unremarkable.

Physical Examination: On examination, he was unresponsive to verbal stimuli and had minimal movement of his limbs to painful stimuli. Pupils were $3 \mathrm{~mm}$ in size, without any reaction to light. Motor testing showed increased muscle tone and there was no preferential movement of any side. Deep tendon reflexes were absent. Plantar stimulation showed extensor responses on both sides. GCS score was 5/15. CT scans of head showed multiple contusions in the frontal, temporal and occipital cerebral regions, brain edema and significant subarachnoid hemorrhage (Figure 1A, 1B and 2).

ICP monitoring on admission showed pressure in the range of $35-40 \mathrm{~mm} \mathrm{H} 2 \mathrm{O}$. He was urgently taken to the $\mathrm{OR}$ and underwent bilateral decompression craniotomies. Follow-up CT scan 12 hours later showed massive brain edema with multiple areas of brain infarctions in spite of decompression (See Figure 3). He succumbed to his injuries 48 hours later.

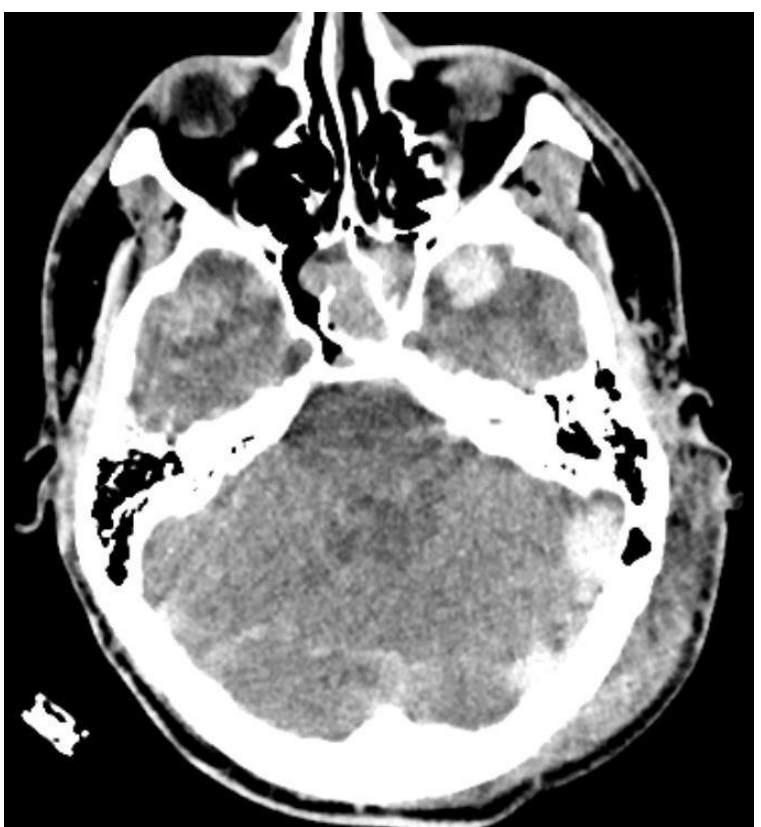

Figure 1A. CT scan of head on day 1 showing temporal and occipital lobe cerebral contusions.

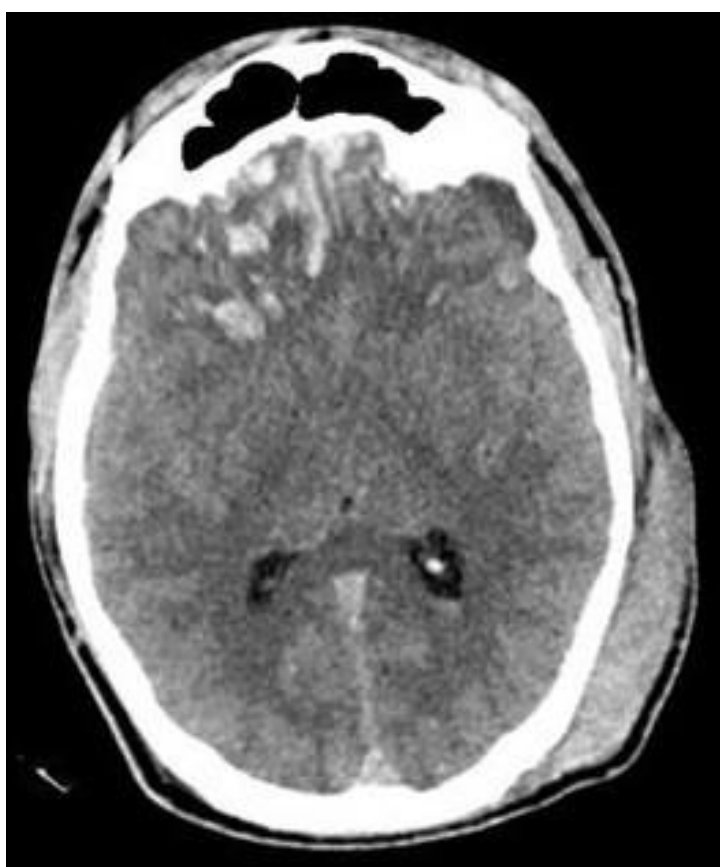

Figure 1B. CT scan of head on day 1 showing right frontal lobe cerebral contusions. 


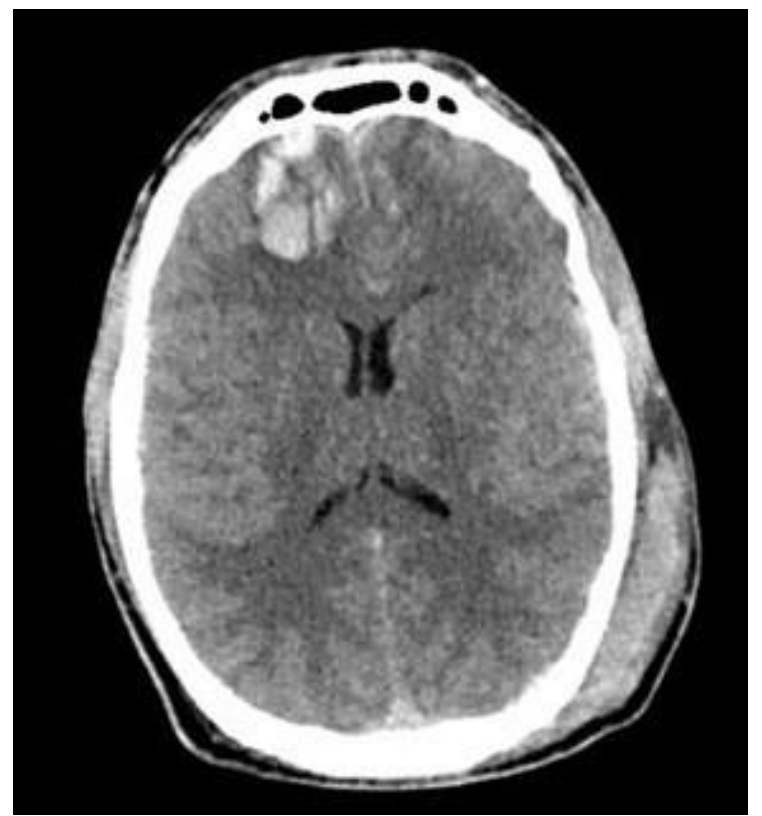

Figure 2. CT scan of head on day 1 showing of right frontal lobe hematoma.

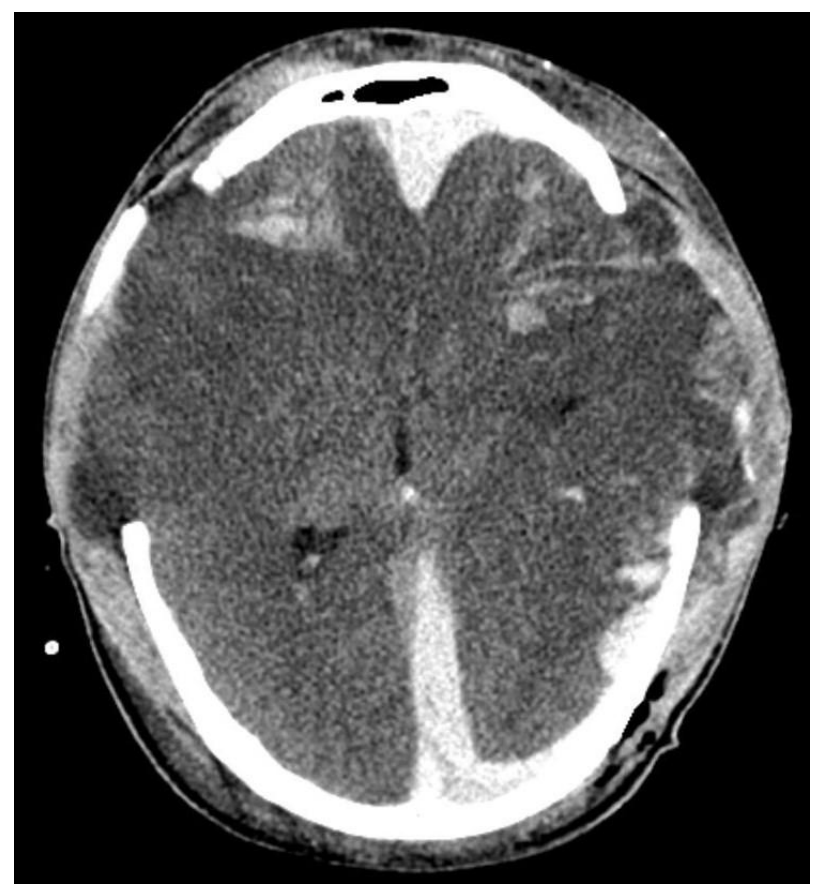

Figure 3. CT scan of head on day 2 showing further evolution of cerebral edema, infarction and bilateral decompression craniotomy.

\section{DisCUSSION}

TBI is one of the major consequences of head trauma that can lead to serious disability or death. With an estimated 10 million cases a year, it is a major public health concern that needs to be addressed. ${ }^{1}$ Among the elderly, falls are one of most common causes of head injury; falls can be due to various factors including alcohol ingestion. ${ }^{2}$ Consumption of alcohol is also a common cause of head injury and fatality in younger age groups.

As demonstrated in the case report, secondary brain injury after alcohol consumption can have deleterious effects. The young male suffered cerebral contusions and brain edema as a result of his injuries sustained after his fall, which became progressively worse ultimately leading to his demise. CT scans are a useful tool which can show a stepwise deterioration of TBI which can be tracked to help in not only diagnosing the extent of injuries but also a patient's prognosis. ${ }^{5}$ Due to the serious consequences of alcohol and its potential for fatal head related injuries, it is important to increase the awareness of this subject among the greater population.

\section{REFERENCES}

1. Wipassakornwarawut S, Suwonmontri P, Thokaow S. Risk factors for mortality in head-injured patients with probability of survival greater than 0.5 . J Med Assoc Thai. 2011 Nov;94(11):1365-721.

2. Kirkpatrick JB, Pearson J. Fatal cerebral injury in the elderly. J Am Geriatr Soc. 1978 Nov;26 (11):489-97

3. Papadopoulos IN, Bonovas S, Kanakaris NK, Nikolopoulos G, Kotsilianou O, Konstantoudakis G, Leukidis C. Alcohol and psychoactive drugs increased the pre-hospital mortality in 655 fall-related fatalities in Greece: A call for management protocols. Injury. 30 December 2010

4. Wang H, Yu X, Xu G, Xu G, Gao G, Xu X. (2011). Alcoholism and traumatic subarachnoid hemorrhage: an experimental study on vascular morphology and biomechanics. J Trauma. Jan;70 (1):E6-12

5. Khan S, Khan N, Masood S, Rana AQ. Evolution of traumatic intracerebral hemorrhage captured with CT imaging: report of a case and the role of serial CT scans. Emerg Radiol. 2010 Nov;17(6):493-6. 